\title{
Vitamin D and Prevention of Cardiovascular Disease and Diabetes:
}

\author{
Why the Evidence Falls Short
}

Sue A. Shapses, PhD and JoAnn E. Manson, MD, DrPH

Department of Nutritional Sciences, Obesity Group, Rutgers University, New Brunswick, New Jersey (Dr Shapses); and Department of Medicine, Brigham and Women's Hospital, Harvard Medical School, Boston, Massachusetts (Dr Manson)

Vitamin D has received widespread attention for its potential role in preventing cardiovascular disease and type 2 diabetes mellitus. Several epidemiological studies have suggested that individuals with low blood levels of vitamin D have increased risks of heart disease, stroke, hypertension, and diabetes. Yet the evidence that vitamin D prevents cardiometabolic outcomes played an inconsequential role in determining the population needs for vitamin D in the 2011 report from the Institute of Medicine (IOM). ${ }^{1}$ How is it possible that such a large body of evidence had so little influence on the recommended intakes for vitamin D? This commentary, authored by 2 members of the IOM Committee, provides a critical appraisal of the relevant evidence, explains why the research was found to be insufficient by the IOM, and identifies future research needs on this topic.

Based on bone health, recommended dietary allowances(covering requirements of $\geq 97.5 \%$ of the population) for vitamin $\mathrm{D}$ are $600 \mathrm{IU} / \mathrm{d}$ for individuals aged 1 to 70 years and 800 IU/d for those older than 70 years, corresponding to a serum 25-hy-droxyvitamin D level of $20 \mathrm{ng} / \mathrm{mL}$ or greater ( $\geq 50 \mathrm{nmol} / \mathrm{L}$ ) under conditions of minimal sun exposure. ${ }^{1}$ The IOM Committee concluded that the evidence that vitamin D prevented cardiovascular disease, diabetes, or other cardiometabolic outcomes (as well as cancer and other nonskeletal outcomes) was inconsistent and inconclusive and did not meet criteria for establishing a cause and effect relationship, which was consistent with an earlier systematic review conducted by the Agency for Healthcare Research and Quality (AHRQ) in 2009. ${ }^{2}$

Most of the available evidence on vitamin D and cardio-metabolic outcomes had been derived from laboratory studies or ecological and observational investigations. ${ }^{1,2}$ Although measures of serum 25-hydroxyvitamin D were considered to be a useful marker of vitamin

(C2011 American Medical Association. All rights reserved.

Corresponding Author: Sue A. Shapses, PhD, Rutgers University, 96 Lipman Dr, New Brunswick, NJ 080901 shapses@aesop.rutgers.edu.

Conflict of Interest Disclosures: Both authors have completed and submitted the ICMJE Form for Disclosure of Potential Conflicts of Interest. Dr Shapses reported that she and her colleagues at Rutgers University are completing a trial funded by grants from the National Institutes of Health examining the effect of vitamin D on calcium absorption (primary analysis) with a secondary analysis of other outcomes including those related to insulin sensitivity. Dr Manson reported that she and her colleagues at Brigham and Women's Hospital, Harvard Medical School, received funding from the National Institutes of Health to conduct the Vitamin D and Omega-3 Trial (VITAL), a large-scale randomized trial of vitamin D and omega-3s in the prevention of cancer and cardiovascular disease. Both authors received reimbursement for travel to Institute of Medicine committee meetings. 
D exposure, correlation between these levels and health outcomes in observational studies does not prove causation, underscoring the need for cautious interpretation. Specific factors relevant to serum 25-hydroxyvitamin D levels are sources of potential confounding for cardiometabolic outcomes, including obesity (due to deposition primarily in adipose tissue), sun exposure, physical activity (correlated with time outdoors), and nutritional status. Other factors such as ethnicity, skin pigmentation, and medications may also affect serum 25hydroxyvitamin D levels.

\section{Cardiovascular Disease}

The vitamin D receptor is expressed in cells throughout the vascular system. Many cell types, including vascular smooth muscle cells, endothelial cells, and cardiomyocytes, produce 1a-hydroxylase, which converts 25 -hydroxyvitamin $\mathrm{D}$ to calcitriol, the natural ligand of the vitamin D receptor. Calcitriol has been shown to inhibit vascular smooth muscle cell proliferation, regulate the renin-angiotensin system, decrease coagulation, and exhibit anti-inflammatory properties.

Ecological studies have suggested higher cardiovascular disease mortality during the winter and in regions with less solar UV-B exposure. Some, but not all, observational studies suggest an inverse association between 25-hydroxyvitamin D levels and clinical cardiovascular disease events. The AHRQ report ${ }^{2}$ identified 4 relevant observational studies. The Framing-ham Offspring Study ${ }^{3}$ and the Health Professionals Follow-up Study ${ }^{4}$ found significant inverse associations between 25-hydroxyvitamin D levels and incident cardiovascular disease events. However, a closer look at the former study indicated that the relationship between 25-hydroxyvitamin $\mathrm{D}$ and cardiovascular disease was nonlinear and reached a plateau between 20 and $30 \mathrm{ng} / \mathrm{mL}$, with a suggestion of slightly increased risk at higher 25-hydroxyvitamin D levels. In addition, the Third National Health and Nutrition Examination Survey (NHANES III) found no significant association between serum 25hydroxyvitamin D and cardiovascular disease mortality, although persons in the lowest quartile had a $26 \%$ increase in total mortality. ${ }^{5}$ The NHANES III analysis suggested a similar U-shaped relationship for 25-hydroxyvita-min $\mathrm{D}$, with increased total mortality not only at low $(<20 \mathrm{ng} / \mathrm{mL})$ but also at high $(>50 \mathrm{ng} / \mathrm{mL})$ levels. Other observational studies have shown mixed results. ${ }^{6}$

There is a paucity of randomized controlled trials of vitamin D and cardiovascular disease events and absence of any trials with cardiovascular disease as the primary prespecifiedoutcome. ${ }^{1,2}$ A British trial that tested $100000 \mathrm{IU}$ of vitamin $\mathrm{D}_{3}$ or placebo every 4 months ( $~ 833 \mathrm{IU} / \mathrm{d})$ for up to 5 years, with cardiovascular disease as a secondary outcome, showed null results. In a small 1-year Australian trial, vitamin D (1000 IU/d) added to calcium supplementation vs calcium alone was associated with a nonsignificantly lower risk of ischemic heart disease events and no difference in the risk of stroke. When data from these 2 trials were combined, the pooled relative risk (RR) for cardiovascular disease was 0.90 (95\% confidence interval [CI], 0.77-1.05) for vitamin D. ${ }^{6}$ In a pooled analysis of 3 trials of combination calcium plus vitamin D vs placebo, including the Women's Health Initiative (WHI), the RR for cardiovascular disease was 1.04 (95\% CI, 0.92-1.18). ${ }^{6}$ Thus, 
the conclusion of both the IOM Committee ${ }^{1}$ and recent systematic reviews ${ }^{2,6}$ was that the evidence was inconsistent and insufficient to prove a cause and effect relationship., ${ }^{2,6}$

\section{Type 2 Diabetes}

The role of calcitriol in the synthesis and secretion of insulin and regulation of calcium trafficking in beta islet cells, and its effects on insulin action have been established in both rodent models and cell culture. ${ }^{1}$

The AHRQ report did not include type 2 diabetes or the metabolic syndrome in its systematic review. ${ }^{2}$ Although some observational studies have shown an inverse relationship between higher vitamin D intake or serum 25-hydroxyvita-min D and risk of type 2 diabetes, a systematic review and meta-analysis ${ }^{7}$ with a large body of observational evidence and 6 intervention studies with vitamin D supplementation found generally neutral results. Studies published after the IOM report ${ }^{1}$ have shown mixed results; there was either a higher risk of prediabetes in individuals (NHANES III) with 25-hydroxyvitamin D levels below $18 \mathrm{ng} / \mathrm{mL}^{8}$ or no association between serum 25-hydroxyvitamin D and risk of type 2 diabetes in a Canadian population or in the WHI.

Randomized trials of vitamin D supplementation and risk of type 2 diabetes have had inconsistent results. In the Rosiglitazone Evaluated for Cardiac Outcomes and Regulation of Glycaemia in Diabetes (RECORD) trial, there was no effect of $800 \mathrm{IU} / \mathrm{d}$ of vitamin $\mathrm{D}_{3}$ supplementation (with or without $1000 \mathrm{mg}$ of calcium carbonate) on incident diabetes over 2 to 5 years (fracture was the primary outcome variable and diabetes outcomes were selfreported). ${ }^{9}$ In separate studies of vitamin D supplementation with $4000 \mathrm{IU} / \mathrm{d}$ or $120000 \mathrm{IU}$ every 2 weeks in South Asian overweight women and obese men, respectively, insulin sensitivity significantly improved compared with placebo. ${ }^{1,2}$ However, another study in overweight adults in Germany found no relationship between vitamin D supplementation (3300 IU/d) with glucose metabolism during weight loss. ${ }^{1}$ In a post hoc analysis of a trial testing the effects of 3 years of supplementation with $700 \mathrm{IU}$ of vitamin D and $500 \mathrm{mg}$ of calcium daily on bone health, individuals with impaired fasting glucose were found to have a lower increase in fasting glucose levels and less insulin resistance compared with placebo controls. ${ }^{1,2}$ In patients with established type 2 diabetes, vitamin $\mathrm{D}$ has not been shown to improve insulin resistance or glucose metabolism. ${ }^{1}$ Thus, the overall evidence from clinical trials and observational research ${ }^{1,2,7-9}$ is insufficient to establish a causal relationship between vitamin D supplementation and type 2 diabetes prevention.

\section{Conclusions}

Despite biological plausibility for a role of vitamin D in the prevention of cardiovascular disease and diabetes, the evidence from available research is inconsistent, inconclusive as to causality, and not sufficient to inform nutritional requirements. No large-scale randomized trials have been completed with cardiovascular disease or diabetes as the primary prespecified outcomes. Although the observational evidence is suggestive of increased risks associated with low levels of serum 25-hydroxyvitamin D, confounding by obesity and behavioral factors cannot be excluded. More research is needed to elucidate whether higher 
intakes of vitamin $\mathrm{D}$ (between the recommended dietary allowance and the tolerable upper intake level) or serum 25 -hydroxyvitamin D levels in the range of 20 to $50 \mathrm{ng} / \mathrm{mL}$ influence cardiovascular disease or diabetes risk. New randomized trials assessing the role of supplementation with vitamin $\mathrm{D}$ in cardiovascular disease and type 2 diabetes prevention are in progress (such as NCT01169259, NCT00736632, and NCT01145703) and should provide important answers within 5 to 6 years. While ongoing and future research may demonstrate clear benefits of vitamin D in relation to cardiometabolic outcomes, and possibly indicate higher intake requirements for this purpose, existing evidence is not yet sufficient to support such conclusions.

\section{References}

1. Institute of Medicine. [Accessed May 20, 2011] Dietary reference intakes for calcium and vitamin D. http://books.nap.edu/openbook.php?record_id=13050

2. Chung, M.; Balk, EM.; Brendel, M., et al. Tufts Evidence-based Practice Center. Vitamin D and Calcium: A Systematic Review of Health Outcomes: Evidence Report No. 183. Rockville, MD: Agency for Healthcare Research and Quality; 2009.

3. Wang TJ, Pencina MJ, Booth SL, et al. Vitamin D deficiency and risk of cardiovascular disease. Circulation. 2008; 117(4):503-511. [PubMed: 18180395]

4. Giovannucci E, Liu Y, Hollis BW, Rimm EB. 25-hydroxyvitamin D and risk of myocardial infarction in men. Arch Intern Med. 2008; 168(11):1174-1180. [PubMed: 18541825]

5. Melamed ML, Michos ED, Post W, Astor B. 25-hydroxyvitamin D levels and the risk of mortality in the general population. Arch Intern Med. 2008; 168 (15):1629-1637. [PubMed: 18695076]

6. Wang L, Manson JE, Song Y, Sesso HD. Systematic review: vitamin D and calcium supplementation in prevention of cardiovascular events. Ann Intern Med. 2010; 152(5):315-323. [PubMed: 20194238]

7. Pittas AG, Lau J, Hu FB, Dawson-Hughes B. The role of vitamin D and calcium in type 2 diabetes. J Clin Endocrinol Metab. 2007; 92(6):2017-2029. [PubMed: 17389701]

8. Shankar A, Sabanayagam C, Kalidindi S. Serum 25-hydroxyvitamin D levels and prediabetes among subjects free of diabetes. Diabetes Care. 2011; 34(5):1114-1119. [PubMed: 21430085]

9. Avenell A, Cook JA, MacLennan GS, McPherson GC. RECORD trial group. Vitamin D supplementation and type 2 diabetes. Age Ageing. 2009; 38(5):606-609. [PubMed: 19617604] 